\title{
Anti-inflammatory effect of stromal vascular fraction cells in fat transplantation
}

\author{
MING ZHU $^{1 *}$, JIAN XUE $^{1 *}$, SHILIANG LU ${ }^{1}$, YI YUAN ${ }^{2}$, YUNJUN LIAO $^{2}$, \\ JINGJING QIU ${ }^{1}$, CHENG LIU ${ }^{1}$ and QINGTING LIAO ${ }^{1}$ \\ ${ }^{1}$ Department of Dermatology, Guangdong Provincial Corps Hospital of Chinese People's Armed Police Forces, \\ Guangzhou Medical University, Guangzhou, Guangdong 510000; ${ }^{2}$ Department of Plastic and Reconstructive Surgery, \\ Nanfang Hospital, Southern Medical University, Guangzhou, Guangdong 510515, P.R. China
}

Received December 13, 2016; Accepted July 30, 2018

DOI: $10.3892 /$ etm.2018.7082

\begin{abstract}
In previous studies, it has been demonstrated that the stromal vascular fraction (SVF) promoted the retention rate of grafts following fat transplantation through its angiogenic properties. However, the effect of SVF on inflammatory response subsequent to fat transplantation remains unclear. Fat from C57 mice were mixed with green fluorescent protein (GFP) SVF cells or phosphate-buffered saline and transplanted into C57BL/6 mice. The graft was harvested and immunofluorescent staining was performed on 1, 7, 14 and 30 days. The numbers of vessels and macrophages, as well as the inflammation level, in the two groups were evaluated. Although the GFP fluorescence indicated that most SVF cells were dead following transplantation, there was a higher graft retention rate in the SVF-assisted group compared with the control group. The SVF-assisted group also presented higher expression levels of interleukin (IL)- 6 and tumor necrosis factor- $\alpha$, and lower levels of IL-10, as well as increased M2 macrophages in comparison with the control group. Therefore, the results indicated that SVF promoted the retention rate of grafts following fat transplantation through the well-known pro-angiogenic mechanism (paracrine function and involvement in the formation of new vessels), as well as through the anti-inflammatory property of SVF (expression and suppression of various cytokines and conversion of the macrophage phenotype).
\end{abstract}

Correspondence to: Dr Ming Zhu, Department of Dermatology, Guangdong Provincial Corps Hospital of Chinese People's Armed Police Forces, Guangzhou Medical University, 268 Yanling Road, Guangzhou, Guangdong 510000, P.R. China

E-mail: 15800031860@163.com

*Contributed equally

Abbreviations: SVF, stromal vascular fraction; GFP, green fluorescent protein; TNF- $\alpha$, tumor necrosis factor- $\alpha$

Key words: stromal vascular fraction, fat transplantation, inflammation, macrophages, cytokines

\section{Introduction}

Fat transplantation has become a tool not only for augmenting tissue volume, but also for enhancing tissue regeneration. With the development of various techniques to assist fat transfer, fat graft retention has become more predictable but is still less than ideal. The stromal vascular fraction (SVF) cells assisted fat transplantation is one of the practical methods to improve fat graft retention. However, the role of SVF in fat grafting is still unclear. To improve these outcomes, it is essential to elucidate the detailed mechanism underlying the engraftment of fat tissue.

SVF is a heterogeneous cell mixture containing all types of fat cells, with the exception of adipocytes (1). Yoshimura et al $(2,3)$ reported that the SVF cell-assisted facial or breast fat transplantation presented better clinical results compared with traditional fat grafting. In addition, our previous study revealed that SVF promoted the fat graft retention rate through the secretion of growth factors, promotion of angiogenesis and increasing the density of stem cells (4). Fat transplantation is a process accompanied by inflammatory cell infiltration and acute inflammatory response (5). Furthermore, several previous studies on diabetes and obesity have suggested that adipose tissue is closely associated with inflammation $(6,7)$. However, the effect of SVF on the inflammatory response following fat transplantation remains unclear.

Therefore, the present study aimed to investigate the effect of SVF on inflammation regulation in the fat transplanted to mice and its impact on fat graft retention in mice.

\section{Materials and methods}

SVF-assisted fat transplantation model. A total of 24 male and 24 female 8-week-old C57BL/6 (Southern Medical University, Guangzhou, China) weighing 20-25 g and 5 male and 5 female green fluorescence protein (GFP) C57BL/6J (Model Animal Research Center of Nanjing University, Nanjing, China) mice were used in the present study. The GFP C57BL/6J mice were 6-8 weeks old and weighed 20-25 g. The GFP C57BL/6J mice were sacrificed to obtain SVF as previously described (8). A total of 24 C57BL/6 mice were sacrificed, and the inguinal fat pads were harvested and dissected into small sections $\left(\sim 1 \mathrm{~mm}^{3}\right)$. 
For fat transplantation, $0.5 \mathrm{ml}$ dissected fat from C57BL/6 mice was mixed with $5 \times 10^{6}$ GFP SVF cells from GFP C57BL/6J mice and suspended in $10 \mu \mathrm{l}$ phosphate-buffered saline (PBS). The mixture was then injected into the subcutaneous tissues on the left flank of C57BL/6 mice using an $1 \mathrm{ml}$ syringe with standardized blunt tipped 14 gauge infiltration cannula, and these mice served as the SVF group $(n=24)$. In the control group, $0.5 \mathrm{ml}$ fat mixed with $10 \mu \mathrm{l}$ PBS was injected into the right flank of the C57BL/6 mice ( $n=24)$. At 1, 7, 14 and 30 days after fat transplantation, the mice were sacrificed and the grafts were harvested and subsequently analyzed as described later. No mortality was observed in the animals following fat transplantation in the present study. All experiments were performed under the approval of the Nanfang Hospital Institutional Animal Care and Use Committee (Guangzhou, China). The volume of the grafts was measured with a displacement method immediately after the grafts were harvested.

Whole-mount staining. Visualization of the fat grafts was performed using the procedure outlined by Eto et al (9). Accordingly, the grafts were cut into 0.5-1-mm sections and incubated with Hoechst 33342 (Sigma-Aldrich; Merck AG, Darmstadt, Germany) for $30 \mathrm{~min}$ at room temperature in order to stain the cell nuclei. The samples were then washed with PBS and observed directly with a confocal microscope system (FV1000 confocal microscope; Olympus Corp., Tokyo, Japan). Hoechst and GFP-positive cells in the SVF group were counted, and the percentage of surviving SVF cells was defined as the number of stained cells with respect to the total number of cells $\left(5 \times 10^{6}\right.$ cells). The number of each cell population per unit volume was then calculated. Images captured from four different fields-of-view for each sample were analyzed.

Histological analysis. Samples were sectioned serially at $5 \mathrm{~mm}$ and stained with hematoxylin and eosin, according to standard protocols. Next, the samples were examined with an Olympus BX51 microscope and images were captured using an Olympus DP71 digital camera (Olympus Corp.).

Immunohistochemical staining. For immunostaining, 5-mm sections were incubated with the rabbit anti-mouse CD31 primary antibody (cat. no. Sc-1506-R; dilution, 1:400; Santa Cruz Biotechnology, Inc., Dallas, TX, USA). The endogenous peroxidase activity was inhibited using $1 \%$ hydrogen peroxide (Rongbai Biological Technology Co., Ltd., Shanghai, China). Following the incubation, the sections were incubated with an EnVision complex (Dako; Agilent Technologies, Inc., Santa Clara, CA, USA) and horseradish peroxidase-conjugated secondary antibody (cat. no. 31460; dilution, 1:5,000; goat anti-rabbit; Gibco; Thermo Fisher Scientific, Inc., Waltham, MA, USA). Color was then developed by 3,3'-diaminobenzidine (Sigma-Aldrich; Merck AG), and CD31-positive cells were visualized and under a biological microscope. The images captured were analyzed with the professional image analysis software IPP (version 6.0; Media Cybernetics, Inc., Rockville, MD, USA). The mean vessel densities were statistically analyzed.

Immunofluorescence staining. Samples cut into 5- $\mu \mathrm{m}$ sections were incubated overnight at $4{ }^{\circ} \mathrm{C}$ with primary antibodies as follows: Rabbit anti-mouse CD206 (cat. no. ab64693; dilution,
1:300; Abcam, Cambridge, UK), and rat anti-mouse Mac2 (cat. no. CL8942G; dilution, 1:200; Cedarlane Corp., Burlington, ON, Canada). Subsequently, samples were incubated at room temperature for $1 \mathrm{~h}$ with the DyLight ${ }^{\circledR} 488$-conjugated goat anti-rabbit immunoglobulin G (cat. no. ab96883; dilution, 1:200; Abcam) and Alexa Fluor 647-conjugated goat anti-rat immunoglobulin G (cat. no. 4418; dilution, 1:200; Cell Signaling Technology, Inc., Danvers, MA, USA) secondary antibodies, correspondingly. Nuclei were stained with DAPI (dilution, 1:200; Sigma-Aldrich; Merck AG) and images were obtained through a confocal microscope system (Zeiss $\mathrm{GmbH}$, Jena, Germany). The number of M1 macrophages (Mac2 ${ }^{+} / \mathrm{CD}_{206}{ }^{-}$) and M2 macrophages (Mac2 ${ }^{+} / \mathrm{CD} 206^{+}$) were counted by analyzing at least six fields of x200 magnification for each sample by Photoshop software (version 7.0; Adobe Systems, Inc., San Jose, CA, USA). Data were collected by two independent, blinded observers.

Enzyme-linked immunosorbent assay (ELISA). Samples were ground to obtain the tissue homogenate, and the supernatant was extracted by centrifugation at $12,000 \mathrm{x} \mathrm{g}$ for $15 \mathrm{~min}$ at $4^{\circ} \mathrm{C}$. The expression levels of interleukin (IL)-6 (cat. no. GTX37124; GeneTex, Inc., Irvine, CA, USA), tumor necrosis factor- $\alpha$ (TNF- $\alpha$; cat. no. 500850; Cayman Chemical, Ann Arbor, MI, USA) and IL-10 (cat. no. GTX37143; GeneTex, Inc.) in the tissue homogenates of the samples were measured using ELISA according to the manufacturers' protocols. The absorbance of the plates was detected on a Multiskan MK3 microplate reader (Thermo Fisher Scientific, Inc.) set at a wavelength of $450 \mathrm{~nm}$.

Statistical analysis. All data are expressed as the mean \pm standard deviations, and the statistical software SPSS version 20.0 (IBM Corp., Armonk, NY, USA) was used for data processing. An independent samples t-test was used to examine differences between the two groups. Analysis of covariance, a general linear model that combines analysis of variance and regression, was used for analysis of different time points within one group. Tukey's test was used as the post hoc test to detect the significant differences between the samples in the same group following the analysis of covariance. $\mathrm{P}<0.05$ was considered to demonstrate differences that were statistically significant.

\section{Results}

Graft volume alterations. The graft volume curves of the two groups at different time points indicated the similar volume tendency of grafts at the initial time points, with the exception of a slight increase in the volume of the control group at day 14 (Fig. 1). The volume presented a marked decline from day 14 after transplantation in the two groups. However, the graft volume of the SVF group was significantly higher compared with that of the control group at day 30 after transplantation $(\mathrm{P}<0.05)$.

Retention rate of GFP SVF cells. The GFP fluorescence in the SVF group was detected in order to reveal the retention rate of SVF cells in the grafts. Quantification of the GFP of SVF cells indicated that the retention rate significantly decreased from $83.5 \pm 10.9 \%$ on day 1 after transplantation to $18.6 \pm 9.0 \%$ 
on day $7(\mathrm{P}<0.05)$, with further decrease observed at later time

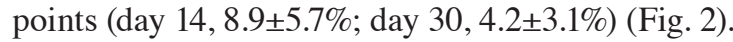

Tissue structure analysis of grafts. Histological observation by hematoxylin and eosin staining demonstrated that an increased number of inflammatory cells infiltrated into the graft in the control group when compared with the SVF group at day 1 after transplantation (Fig. 3). At days 7 and 14, the number of inflammatory cells decreased, however, the number of new vessels increased in the two groups. The SVF group presented increased number of small-sized adipocytes compared with the control group at days 7 and 14 , as well as a more mature structure of the adipose tissue at day 30 (Fig. 3).

Vascular alterations of the grafts. In order to observe the vascular alterations of the fat grafts in more detail, immunostaining of vascular endothelial cells with CD31 antibody was conducted. As shown in Fig. 4, the vessel density of the SVF group was significantly higher compared with that in the control group at days 7, 14 and 30 after transplantation $(\mathrm{P}<0.05)$.

Infiltration of M2 macrophages. The immunofluorescence analysis demonstrated the infiltration of M1 macrophages (Mac2 ${ }^{+} / \mathrm{CD} 206 ;$; red fluorescence) and M2 macrophages (Mac2 ${ }^{+} / \mathrm{CD} 206^{+}$; purple fluorescence) in the two groups days 7 after transplantation (Fig. 5A). The ratio of M1/M2 was significantly higher in the control group at day 7 compared with the SVF group $(\mathrm{P}<0.05)$ (Fig. 5B). By contrast, the number of M1 and M2 macrophages was relatively similar in the SVF group at day 7, as well as in the control group at day 14, as observed by the ratio of M1/M2. In addition, the SVF group presented a significantly lower ratio of M1/M2 at day 14 compared with the control group $(\mathrm{P}<0.05)$.

Expression levels of pro-inflammatory (IL- 6 and TNF- $\alpha$ ) and anti-inflammatory (IL-10) cytokines. ELISA was used to detect the expression levels of the pro-inflammatory IL-6 and TNF- $\alpha$ cytokines, as well as of the anti-inflammatory IL-10 cytokine. The expression of IL-6 in the two groups increased between days 1 and 14, and then decreased on day 30, with the exception of the IL-6 level in the control group that presented a slight decrease between days 7 and 14 (Fig. 6A). In addition, the expression of TNF- $\alpha$ in the two groups was maintained at a high level prior to day 7 and then decreased on days 14 and 30 (Fig. 6B). Notably, statistical analysis demonstrated that the SVF group had a significantly lower expression level of IL-6 on days 7, 14 and 30 compared with the control, while the TNF- $\alpha$ levels were also significantly decreased in the SVF group on days 1,7 and $14(\mathrm{P}<0.05)$ (Fig. 6A and B). By contrast, compared with the control group, the SVF group presented a markedly higher expression level of IL-10 at days 7 and 14 $(\mathrm{P}<0.05)$ (Fig. 6C).

\section{Discussion}

At the early stage of fat transplantation, acute ischemia and inflammation occur due to inadequate blood supply and tissue injury, which leads to tissue edema (10). It is hypothesized that this may cause the increase in the graft volume observed in the control group at day 14 in the present study. By contrast, the SVF

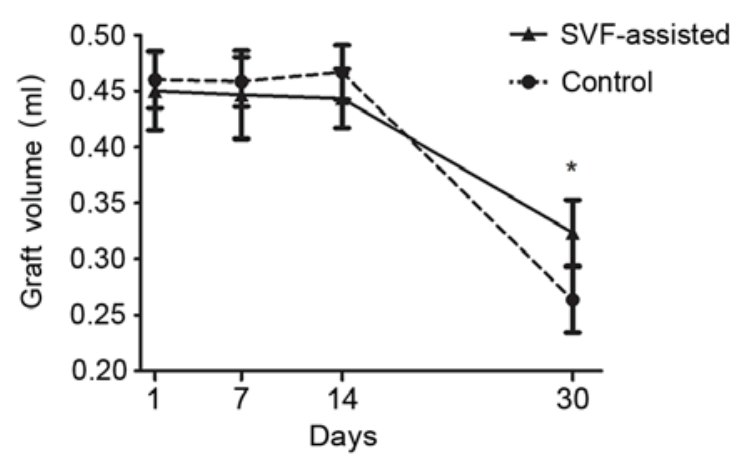

Figure 1. Fat graft volumes in the two groups at different time points. The volume of the grafts presented an evident decline after day 14 following transplantation in the two groups. However, the graft volume in the SVF group was significantly higher compared with the control group at day 30 . ${ }^{*} \mathrm{P}<0.05$ vs. control group. SVF, stromal vascular fraction.

group did not present a graft volume increase, indicating that there was a lower level of edema as a result of better blood supply and lower inflammation compared with the control group.

SVF has been applied in the treatment of ischemia diseases and burn wounds due to its ability to promote angiogenesis and neovascularization $(11,12)$. In our previous animal study it was demonstrated that SVF cell-assisted fat transplantation in nude mice resulted in a higher graft retention rate with enhanced angiogenesis (4). In the present study, it was noted that the vascular network exhibited more rapid growth in the SVF group in comparison with the control group, which gradually grew into the center of the graft from the peripheral region in the SVF group. Furthermore, the SVF group demonstrated higher vessel density compared with the control group. This sufficient blood supply in the SVF group significantly contributed to the improved transplantation results.

The process of angiogenesis is regulated by various growth factors, including vascular endothelial growth factor (VEGF), basic fibroblast growth factor (bFGF), hepatocyte growth factor and transforming growth factor- $\beta$. Our previous study indicated a significant increase of VEGF and bFGF levels in the SVF group, and suggested that SVF cells had a strong paracrine function (4). Several other studies demonstrated that the SVF cells, including endothelial progenitor cells (EPCs), adipose-derived stem cells (ASCs), macrophages and fibroblasts, are able to secret various angiogenic growth factors $(1-3,9)$.

Besides the paracrine effect, the cellular components of the SVF, such as the endothelial cells and pericytes, directly reassemble to form new vessels (4). SVF is a source of progenitor and stem cells, which have the potential to differentiate along different lineages (4). The key component of the SVF is ASCs, identified as CD $34^{+} \mathrm{CD} 31^{-} \mathrm{CD} 45^{-}$, which differentiate into osteogenic, adipogenic and chondrogenic cell types (4). Other important subtypes with progenitor ability include the EPCs and pericytes (9). These cells possess a high proliferative potential and are also able to differentiate into vascular cells, participating in the formation of the new vascular network (1).

In the present study, the SVF group demonstrated reduced level of inflammation compared with the control group, as observed by the lower expression of inflammatory cytokines, including IL- 6 and TNF- $\alpha$, as well as the higher expression 


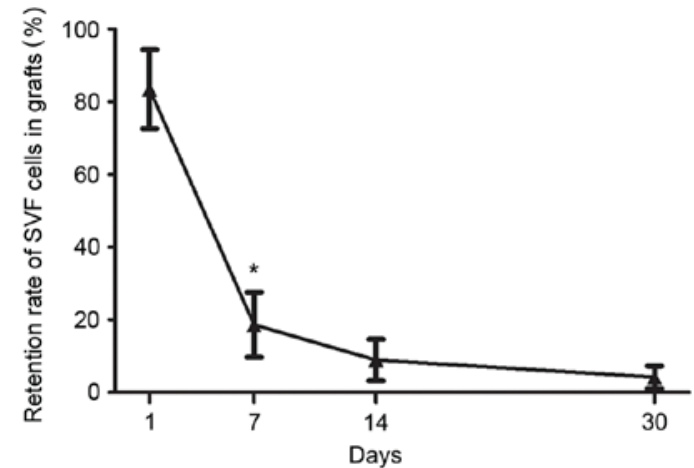

Figure 2. Quantification of surviving SVF cells ( $n=6$ ) following SVF-assisted fat transplantation. The total retention rate of SVF cells decreased sharply at day 1 , followed by a further decline over the subsequent time points. ${ }^{*} \mathrm{P}<0.05$ vs. day 1 . SVF, stromal vascular fraction.

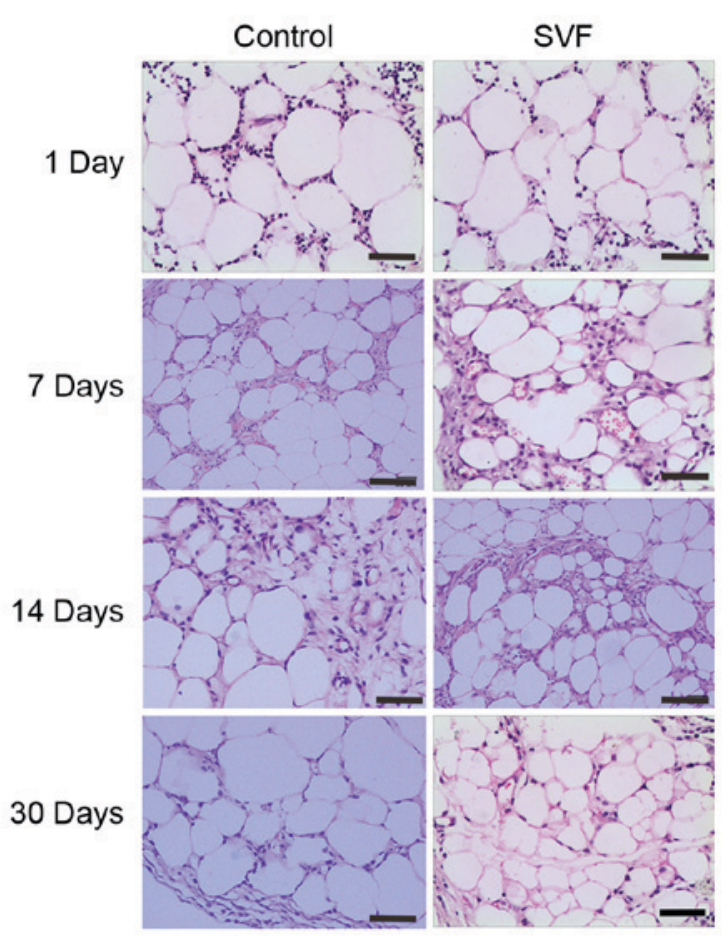

Figure 3. Histological changes of the fat grafts in the SVF and control groups, observed by hematoxylin and eosin staining at different time points. More inflammatory cells infiltrated into the graft in the control group compared with the SVF group at day 1 after transplantation. At days 7 and 14, the number of inflammatory cells decreased, although the number of new vessels increased in the two groups. The SVF group demonstrated an increased number of small-sized adipocytes compared with the control group at days 7 and 14 , as well as a more mature structure of adipose tissue at day 30 . Scale bars, $100 \mu \mathrm{m}$. SVF, stromal vascular fraction.

of anti-inflammatory cytokines, such as IL-10. Furthermore, an increased number of M2 macrophages were detected in the SVF group in comparison with the control group. Tiemessen et al (13) indicated that T-regulatory cells contained in the SVF maintained the M2 phenotype of the macrophages in the SVF. A previous study on ischemia heart failure also revealed that SVF transplantation served an anti-inflammation role in cell therapy through the decrease of myocardial mRNA expression of the inflammatory cytokines TNF- $\alpha$, IL-6, matrix metalloproteinase (MMP-1) and tissue inhibitor

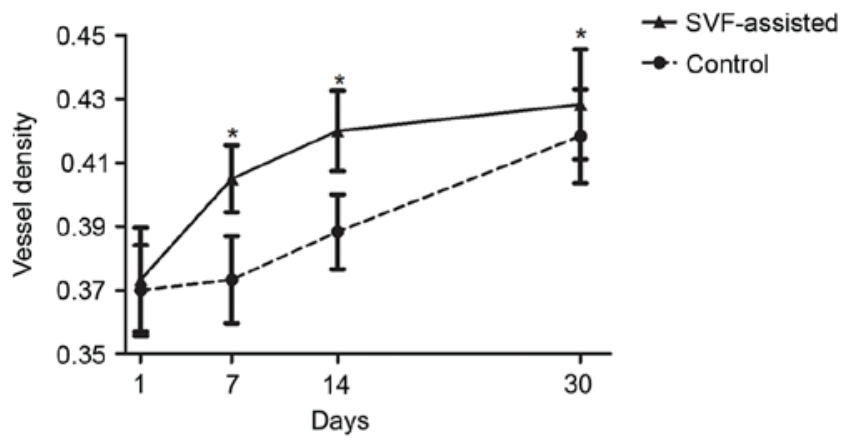

Figure 4. Immunohistochemical staining with CD31 was conducted to observe the vessels of the fat grafts. The vessel density in the SVF group was higher compared with that in the control group at days 7,14 and 30 days. ${ }^{*} \mathrm{P}<0.05$ vs. control group. SVF, stromal vascular fraction.

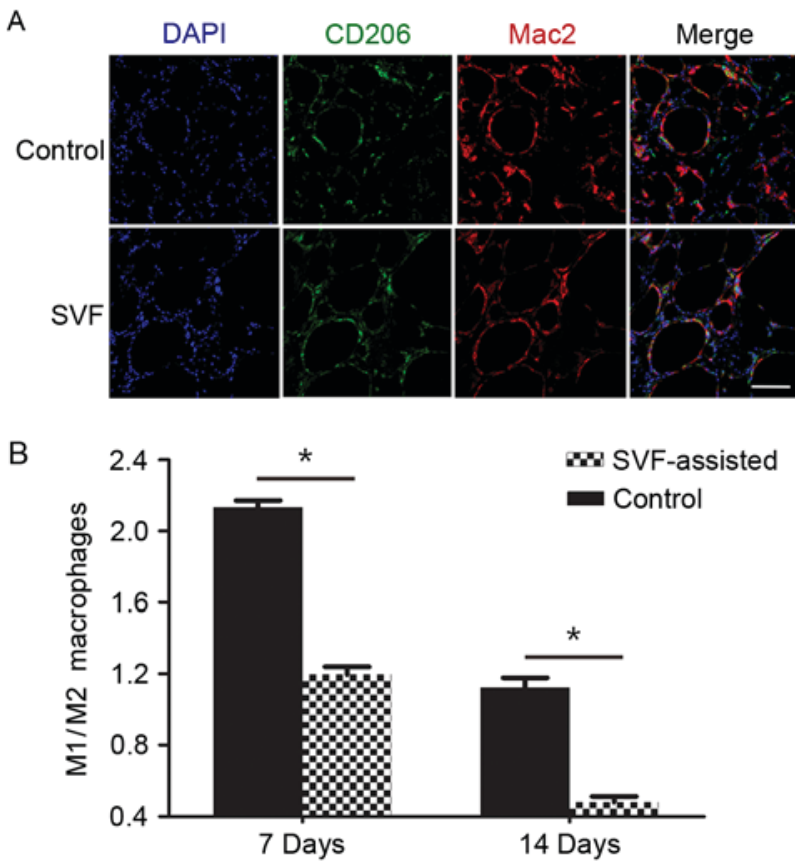

Figure 5. Immunofluorescence staining with CD206 and Mac2 antibodies was performed to observe the macrophages of the fat grafts. (A) Grafts harvested in the control and SVF groups at day 7 after transplantation were stained with DAPI (cell nuclei staining; blue), CD206 (green) and Mac2 (red). The M1 macrophages (Mac2 $2^{+} / \mathrm{CD} 206$; red) and M2 macrophages (Mac2 $2^{+} / \mathrm{CD} 206^{+}$; purple) were observed in the merge images. Scale bar, $50 \mu \mathrm{m}$. (B) M1/M2 macrophage ratio is shown. A high ratio was observed in the control group at day 7, whereas the number of M1 and M2 macrophages was similar in the SVF group at day 7 (ratio close to 1), as well as in the control group at day 14 . The SVF group presented lower ratio of M1/M2 compared with the control group at day $14 .{ }^{*} \mathrm{P}<0.05$. SVF, stromal vascular fraction.

of MMP (TIMP-1) (11). Therefore, in the current study, it is hypothesized that SVF presented anti-inflammatory properties following fat transplantation through the expression and suppression of various cytokines, as well as the conversion of the macrophage phenotype.

The improved graft results observed in the SVF group were due to not only the earlier and sufficient angiogenesis, but also the lower level of inflammation. The damage and ischemia subsequent to fat transplantation lead to inflammatory response, which results in increased cell death, 

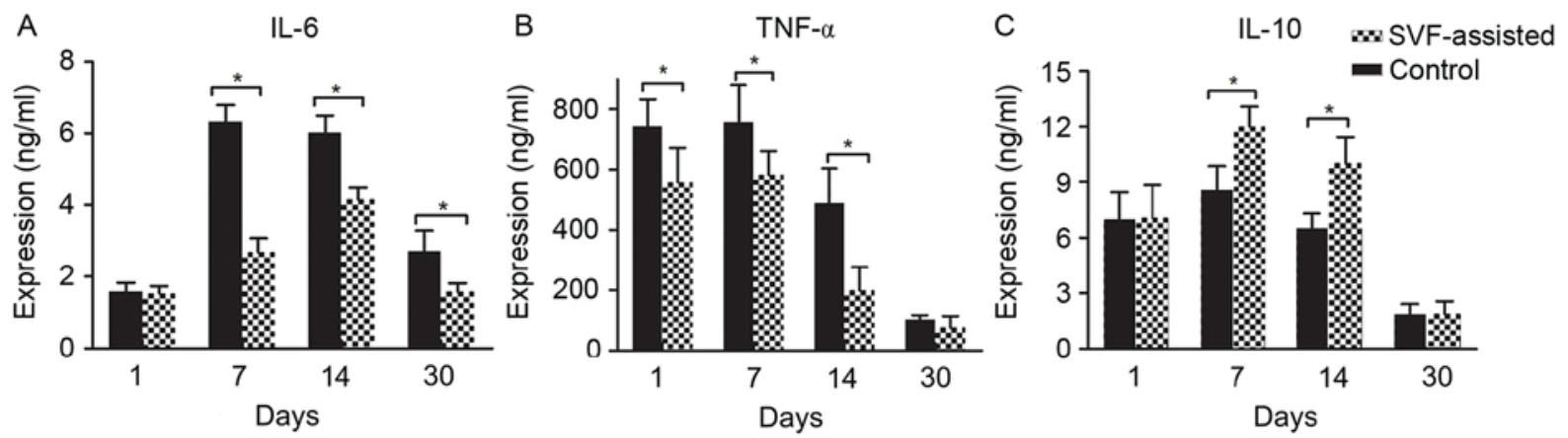

Figure 6. Expression levels of (A) IL-6, (B) TNF and (C) IL-10 of fat grafts, evaluated by enzyme-linked immunosorbent assay. "P<0.05. IL, interleukin; TNF- $\alpha$, tumor necrosis factor- $\alpha$; SVF, stromal vascular fraction.

although inflammation also contributes to angiogenesis (10). However, the improved angiogenesis in the SVF group in the current study demonstrated that the effect of SVF in directly promoting angiogenesis counteracted its indirect inhibition of inflammation that suppressed angiogenesis.

In conclusion, the results of the present study indicated that SVF promoted the retention rate of grafts following fat transplantation through the well-known pro-angiogenic mechanism (paracrine function and involvement in the formation of new vessels), as well as through the anti-inflammatory properties of SVF, which was observed by the expression and suppression of various cytokines, and the conversion of the macrophage phenotype.

\section{Acknowledgements}

Not applicable.

\section{Funding}

The current study was funded by the Nature Science Foundation of Guangdong Province (grant no. 2014A030310458).

\section{Availability of data and materials}

All data generated or analyzed during this study are included in this published article.

\section{Authors' contributions}

MZ, JX, SL, YY and YL designed the study, performed the experiments, analyzed the data, and wrote the paper. JQ, CL and QL collected the data. All authors read and approved the final manuscript.

\section{Ethics approval and consent to participate}

All experiments were performed under the approval of the Nanfang Hospital Institutional Animal Care and Use Committee.

\section{Patient consent for publication}

Not applicable.

\section{Competing interests}

The authors have declared no conflicting interests.

\section{References}

1. Yoshimura K, Suga HandEto H: Adipose-derived stem/progenitor cells: Roles in adipose tissue remodeling and potential use for soft tissue augmentation. Regen Med 4: 265-273, 2009.

2. Yoshimura K, Sato K, Aoi N, Kurita M, Inoue K, Suga H, Eto H, Kato H, Hirohi T and Harii K: Cell-assisted lipotransfer for facial lipoatrophy: Efficacy of clinical use of adipose-derived stem cells. Dermatol Surg 34: 1178-1185, 2008.

3. Yoshimura K, Sato K, Aoi N, Kurita M, Hirohi T and Harii K: Cell-assisted lipotransfer for cosmetic breast augmentation: Supportive use of adipose-derived stem/stromal cells. Aesthetic Plast Surg 32: 48-57, 2008.

4. Zhu M, Dong Z, Gao J, Liao Y, Xue J, Yuan Y, Liu L, Chang Q and Lu F: Adipocyte regeneration after free fat transplantation: Promotion by stromal vascular fraction cells. Cell Transplant 24: 49-62, 2015.

5. Dong Z, Fu R, Liu L and Lu F: Stromal vascular fraction (SVF) cells enhance long-term survival of autologous fat grafting through the facilitation of M2 macrophages. Cell Biol Int 37: 855-859, 2013.

6. Hocking SL, Stewart RL, Brandon AE, Suryana E, Stuart E, Baldwin EM, Kolumam GA, Modrusan Z, Junutula JR, Gunton JE, et al: Subcutaneous fat transplantation alleviates diet-induced glucose intolerance and inflammation in mice. Diabetologia 58: 1587-1600, 2015.

7. Tran TT, Yamamoto Y, Gesta S and Kahn CR: Transplantation of subcutaneous fat to the visceral cavity induced protective metabolic effects: Evidence for intrinsic properties of subcutaneous fat. Diabetes 56 :A5-A6, 2007.

8. Zhang S, Dong Z, Peng Z and Lu F: Anti-aging effect of adipose-derived stem cells in a mouse model of skin aging induced by D-galactose. PLoS One 9: e97573, 2014.

9. Eto H, Suga H, Matsumoto D, Inoue K, Aoi N, Kato H, Araki J and Yoshimura K: Characterization of structure and cellular components of aspirated and excised adipose tissue. Plast Reconstr Surg 124: 1087-1097, 2009

10. Eto H, Kato H, Suga H, Aoi N, Doi K, Kuno S and Yoshimura K: The fate of adipocytes after nonvascularized fat grafting: Evidence of early death and replacement of adipocytes. Plast Reconstr Surg 129: 1081-1092, 2012.

11. Premaratne GU, Ma LP, Fujita M, Lin X, Bollano E and Fu M: Stromal vascular fraction transplantation as an alternative therapy for ischemic heart failure: Anti-inflammatory role. J Cardiothorac Surg 6: 43, 2011.

12. Ceserani V, Ferri A, Berenzi A, Benetti A, Ciusani E, Pascucci L, Bazzucchi C, Coccè V, Bonomi A, Pessina A, et al: Angiogenic and anti-inflammatory properties of micro-fragmented fat tissue and its derived mesenchymal stromal cells. Vasc Cell 8: 3, 2016.

13. Tiemessen MM, Jagger AL, Evans HG, van Herwijnen MJ, John S and Taams LS: CD4+CD25+Foxp3+ regulatory T cells induce alternative activation of human monocytes/macrophages. Proc Natl Acad Sci USA 104: 19446-19451, 2007. 